\title{
Talla, peso y perímetro craneano según edad gestacional en recién nacidos de menos de 35 semanas
}

\author{
Dr. Juan Pablo Beca I. ${ }^{1}$; Dra. Mafalda Rizzardini P. ${ }^{2}$; Dra. Elizabeth Weidt S. ${ }^{3}$ \\ Dra. Maria del Pilar Fernández F. ${ }^{4}$; Dra. Janet Bloomfield G. ${ }^{5}$; \\ Dr. Sergio Corralán V. \\ Weight, height and head circumference in \\ newborns under 35 weeks gestational age
}

\begin{abstract}
A retrospective and colaborative study was done in Santiago, Chile, in order to obtain national data on birthweight, height and head circumference of babies born at 24 to 34 weeks of gestation: 370 babies with reliable gestational age, single pregnancies and no maternal nor fetal morbidity were included in the study. Babies were born in three goverment and one private hospitals from 1982 to 1987 . Mean birthweight, height and head circumference for each gestational age from 24 to 34 weeks are presented in tables with their S.D. and charts \pm 1.5 S.D. The national use of these tables and curves is recommended.

(Key words: low birth weight, growth, gestational age).
\end{abstract}

Tanto el tratamiento como la prevención de parte importante de la patología del recién nacido se basa en un adecuado diagnóstico de la edad gestacional y del estado nutritivo. Para tal efecto, desde la publicación de las clásicas curvas de Lubchenco ${ }^{1,2}$ se clasifica a los R.N. según su peso en relación a la edad gestacional en adecuados, grandes o pequeños para su edad. Sin embargo, se ha visto que estas curvas, basadas en el estudio de recién nacidos en Denver, reflejan una realidad local que no siempre se ha reproducido. Por tal razón, han aparecido en la literatura numerosas curvas de crecimiento intrauterino, que por lo general difieren de las de Lubchenco en las edades gestacionales mayores $s^{3-35}$.

En nuestro medio se han publicado varias tablas de peso $y$ edad gestaciona $1^{12-14}$ que al igual que las anteriores muestran en edades gestacionales mayores, pesos de nacimiento superiores a los de las Curvas de Denver. Sin embargo, todas estas publicaciones carecen de

1. Clínica Alemana de Santiago.

2. Hospital Roberto del Río.

3. Maternidad Hospital del Salvador.

4. Maternidad José Joaquín Aguirre.

5. Clínica Alemana de Santiago.

6. Hospital Dr. Sótero del Río. un número adecuado de recién nacidos de menos de 35 semanas y no contamos, por lo tanto, con datos nacionales para estas edades.

Con el propósito de construir curvas de crecimiento por edad gestacional en recién nacidos chilenos entre 24 y 34 semanas de gestación se realizó un estudio retrospectivo colaborativo en los Servicios de Neonatología del Hospital José Joaquin Aguirre, Maternidad del Hospital del Salvador, Hospital Sótero del Río y de la Clínica Alemana de Santiago.

\section{MATERIAL Y METODO}

Se ingresaron a este estudjo 379 recién nacidos de menos de 35 semanas de gestación nacidos en Santiago, a 540 metros sobre el nivel del mar, entre 1982 y 1987. De ellos, 82 nacjeron en el Hospital José Joaquín Aguirre; 191 en el Hospital del Salvador; 15 en el Hospital Sótero del Río, y 91 en la Clínica Alemana. Se revizaron las fichas clínicas de los períodos correspon. dientes y se consideraron para el estudio sólo aquellos casos que cumplieton con bs requisitos previamente establecidos. De tal manera que los 379 casos estudiados corresponden a una proporción de los casos inicjalmente revisados que, de uno a otro centro, varió entre 35 y $42 \%$.

Los recién racidos admitidos en el estudio fueron aquellos que nacieron de embarazos controlados, oon fecha de última menstruación confiable en la ficha clínica, cálculo pedíttrico de edad gestacional concordante $y$ ausencia de enfermedad fetal o materna. 
Como enfermedad fetal se consideraron causales de exclusión del estudio las infecciones prenatales, malformaciones mayores, genopatías y otras condiciones que pudieran haber alterado el crecimiento fetal. Las afecciones del embarazo que motivaron exclusión del estudio fueron colestasia intrahepática del embarazo con ictericia, madres $R$ h negativas sensibilizadas, diabetes materna, hipertensión arterial crónica, cardiopatías con insuficiencia cardiaca, nefropatías con infección crónica del tracto urinario, rotura de membranas de más de 6 días, metrorragias del segundo o tercer trimestre, alteraciones placentarias crónicas y embarazos múltiples.

La edad gestacional se consideró confiable cuando la edad cronológica, basada en la fecha de la última menstruación, coincidió con la estimación clínica de edad hecha por el neonatólogo con uлa variación de no más de una semana.

Los datos de los 379 recién nacidos estudiados se ingresaron en una ficha indjvidual de estudio $y$ se tabularon por edad gestacional y procedencia. Al no haber diferencia por sexo ni entre los distintos centros, los datos se sumaron por grupos de edad gestacional.

Las medidas fueron tomadas antes de las 24 horas de vida. El peso se determinó en el momento de nacer con el cordón fresco cortado a más o menos $4 \mathrm{~cm}$ de ia superficie abdominal y anudado con huincha elástica. La talla se midió con infantómetro en decúbito dorsal con el pie en ángulo recto sobre la superficie tope inferior y el perímetio cefálico se midió sobre el occipucio y la región superciliar.

Se calcularon los promedios y desviación estándar para peso, talla y circunferencia de cráneo para cada edad gestacional entre 24 y 34 semanas. Para la confección de las respectivas curvas se prefirió emplear el promedio $\pm 1,5$ desviaciones estánáa (DE) lo que incluye a $86 \%$ de la población. Las curvas fueron debidamente suavizadas.

\section{RESULTADOS}

El número de niños estudiadios de cada edad gestacional entre las 24 y las 34 semanas, su promedio de peso y su variación más y menos 1 y 1,5 desviaciones estándar aparecen en la tabla 1 .

El promedio de talla por edad gestacional entre las 24 y las 34 semanas y su variación más y menos 1 y 1,5 desviaciones estándar aparecen en la tabla 2.

El promedio de perímetro craneano para cada edad gestacional entre las 24 y las 34 semanas de gestación, asi como su variación más y menos 1 y 1,5 desviaciones estándar aparecen en la tabia 3 .

Las curvas de peso, talla y circunferencia de cráneo entre las 24 y las 34 semanas de edad gestacional, incluyendo el promedio $\pm 1,5$ desviación estándar, debidamente suavizadas aparecen en las figuras 1,2 y 3 .

\section{DISCUSION}

Existen numerosos factores que reconocidamente influyen sobre el crecimiento del feto. Los más importantes parecen ser: el patrimonio genético, el micro y el macroambiente, $y$, según algunos ${ }^{16}$, la capacidad de adaptación del feto a los factores adversos que lo rodean como la altura sobre el nivel del mar que parece ejercer influencia importante en el último trimestre del embarazol, 9, 17 .

Cuando en 1963 Lubchenco y cols. comunicaron la Curva de Crecimiento Intrauterino de recién nacidos en Denver, se pensó que este patrón de crecimiento podría ser aplicado en cualquier localidad y que de haber diferencias éstas debieran ser atributbles a factores ambientales susceptibles de ser modificados. Más tarde

Tabla 1

Peso por edad gestacional

\begin{tabular}{crrrrrrrr}
\hline Semanas & $\mathbf{n}$ & $\overline{\mathrm{X}}$ & $\mathrm{DE}$ & $-1,5 \mathrm{DE}$ & $-1 \mathrm{DE}$ & $\overline{\mathrm{X}}$ & $+\mathbf{1 D E}$ & $+1,5 \mathrm{DE}$ \\
\hline 24 & 11 & 755 & $\mathbf{1 3 8}$ & 548 & 617 & 755 & 893 & 962 \\
25 & 16 & $\mathbf{8 5 6}$ & $\mathbf{1 4 5}$ & 639 & 711 & 856 & 1.001 & $\mathbf{1 . 0 7 4}$ \\
26 & 23 & 936 & $\mathbf{1 6 9}$ & 683 & 767 & 936 & 1.105 & $\mathbf{1 . 1 9 0}$ \\
27 & 24 & 1.036 & 139 & 828 & $\mathbf{8 9 7}$ & 1.036 & 1.175 & $\mathbf{1 . 2 4 5}$ \\
28 & 29 & 1.179 & 193 & 890 & 986 & 1.179 & 1.372 & $\mathbf{1 . 4 6 9}$ \\
29 & 19 & 1.282 & 164 & 1.036 & 1.118 & 1.281 & 1.446 & 1.528 \\
30 & 34 & 1.476 & 282 & 1.053 & 1.194 & 1.476 & 1.758 & $\mathbf{1 . 8 9 9}$ \\
31 & 43 & 1.628 & 227 & 1.288 & 1.401 & 1.628 & $\mathbf{1 . 8 5 5}$ & $\mathbf{1 . 9 6 9}$ \\
32 & 52 & 1.850 & 282 & 1.427 & 1568 & 1.850 & $\mathbf{2 . 1 3 2}$ & 2.273 \\
33 & 54 & 2.040 & 329 & 1.547 & 1.711 & 2.040 & 2.369 & 2.534 \\
34 & 74 & 2.169 & 193 & 1.880 & 1.976 & 2.169 & 2.362 & 2.459 \\
\hline
\end{tabular}


Tabla 2

Talla por edad gestacional

\begin{tabular}{cccccccc}
\hline Semanas & $\overline{\mathrm{X}}$ & $\mathrm{DE}$ & $-1,5 \mathrm{DE}$ & $-1 \mathrm{DE}$ & $\overline{\mathrm{X}}$ & $+1 \mathrm{DE}$ & $+1,5 \mathrm{DE}$ \\
\hline 24 & 32,7 & 2.24 & 29,3 & 30,4 & 32,7 & $\mathbf{3 4 , 9}$ & $\mathbf{3 6 , 0}$ \\
25 & 33,2 & 2,71 & 29,1 & 30,5 & 33,2 & 35,9 & 37,3 \\
26 & 34,6 & 3,18 & 29,8 & 31,4 & 34,6 & 37,8 & 39,4 \\
27 & 35,2 & 2,31 & 31,8 & 32,9 & 35,2 & 37,6 & 38,7 \\
28 & 37,0 & 3,88 & 31,2 & $33, \mathbf{1}$ & 37,0 & 40,9 & 42,8 \\
29 & 38,5 & 2,04 & 35,5 & 36,5 & 38,5 & 40,6 & 41,6 \\
30 & 39,1 & 3,92 & 33,3 & 35,2 & 39,1 & 43,1 & 45,0 \\
31 & 41,2 & 2,16 & 38,0 & 39,1 & 41,2 & 43,2 & 44,5 \\
32 & 42,3 & 2,22 & 39,0 & 40,1 & 42,3 & $\mathbf{4 4 , 5}$ & 45,6 \\
33 & 43,9 & 2,01 & 40,9 & 41,9 & 43,9 & 45,9 & 46,9 \\
34 & 44,5 & 2,48 & 40,8 & 42,1 & 44,5 & 47,0 & 48,3 \\
\hline
\end{tabular}

Tabla 3

Perímetro craneano pot EG

\begin{tabular}{cccccccc}
\hline Semanas & $\bar{X}$ & $\mathrm{DE}$ & $-1,5 \mathrm{DE}$ & $-1 \mathrm{DE}$ & $\overline{\mathrm{x}}$ & $+1 \mathrm{DE}$ & $+1,5 \mathrm{DE}$ \\
\hline 24 & 23,3 & 1,0 & 21,8 & 22,3 & 23,3 & $\mathbf{2 4 , 3}$ & $\mathbf{2 4 , 8}$ \\
25 & 23,3 & 1,3 & 21,4 & 22,0 & 23,3 & $\mathbf{2 4 , 6}$ & 25,3 \\
26 & 24,9 & 1,9 & 22,1 & 23,0 & 24,9 & $\mathbf{2 6 , 8}$ & 27,8 \\
27 & 25,6 & 1,5 & 23,4 & 24,1 & 25,6 & 27,1 & 27,9 \\
28 & 26,9 & 2,1 & 23,8 & 24,8 & 26,9 & 29,0 & 30,1 \\
29 & 27,0 & 1,3 & 25,1 & 25,7 & 27,0 & $\mathbf{2 8 , 3}$ & 29,0 \\
30 & 28,4 & 1,5 & 26,2 & 26,9 & 28,4 & 29,9 & 30,7 \\
31 & 29,1 & 1,6 & 26,7 & 27,5 & 29,1 & 30,7 & 31,5 \\
32 & 30,5 & 2,4 & 26,9 & 28,1 & 30,5 & 32,9 & 34,1 \\
33 & 31,0 & 1,4 & 28,9 & 29,6 & 31,0 & 32,4 & 33,1 \\
34 & 31,7 & 1,4 & 29,6 & 30,3 & 31,7 & 33,1 & 33,8 \\
\hline
\end{tabular}

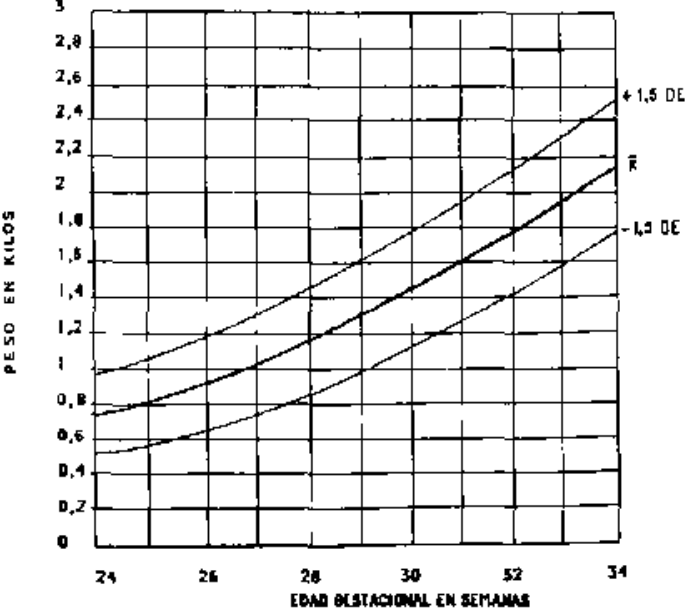

Figura 1: Curva de peso de nacimiento por edad gestacional en recién nacidos chilenos entre 24 y 34 semanas. Promedio $\pm 1,5 \mathrm{DE}$.

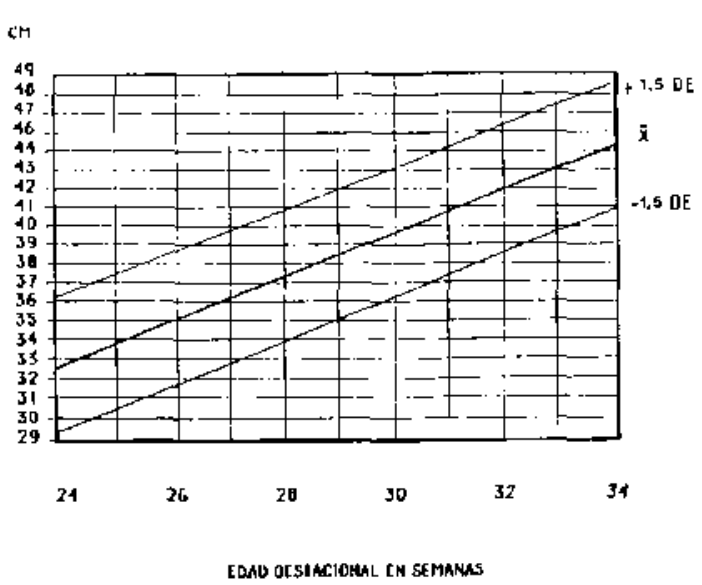

Figura 2: Talla al nacer por edad gestacional en recién nacidos chilenos entre 24 y 34 semanas. Promedio $\pm 1,5 \mathrm{DE}$. 


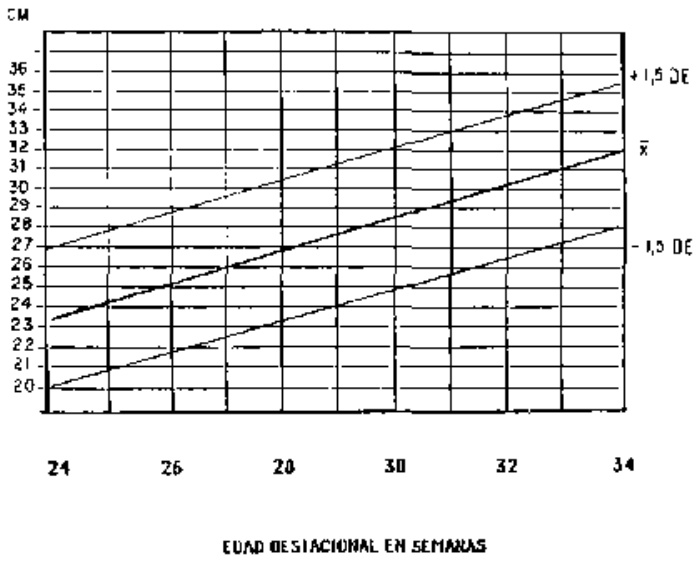

Figura 3: Perímetro craneano al nacer por edad gestacional en recién nacidos chilenos entre 24 y 34 semanas. Promedio $\pm 1,5$ DE.

han aparecido numerosas otras curvas y en todas se observa que en los dos primeros trimestres del embarazo el peso promedio para cada edad es muy semejante. No parece influir en este período el $\operatorname{sexo}^{3,6}$, la raza ${ }^{4}$, la altura $a^{1,9,11,17}$ ni el nivel socioeconómico ${ }^{\mathrm{I}}$. Después de las 36 a 37 semanas, sin embargo, las diferencias entre uno $y$ otro patrón son a veces muy sig. nificativas $5,6,18$.

Las curvas ideales de crecimiento fetal debieran establecerse en estudios prospectivos con edad gestacional conocida mediante ecografia antes de las 12 semanas, en embarazos sin patolo. gía y con controles seriados que aseguren la continuidad del crecimiento fetal. Las curvas actualmente disponibles carecen de esta precisión $\mathrm{y}$ todas tienen márgenes de error en la determinación de la edad gestacional que pueden influir en los resultados. Los resultados de este estudio tienen igual limitación y pueden ser considerados un instrumento válido para ser usado mientras no se cuente con curvas más exactas, con número suficiente de niffos en cada edad gestacional.

Los estudios nacionales ${ }^{12,13,14}$, al igual que numerosos estudios extranjeros ${ }^{3-15}$, han mostrado para edades gestacionales de 35 y más semanas pesos de nacimiento más altos que los de las curvas de Denver. Para edades gestacionales menores, sin embargo, no se habían publicado datos suficientes en el país. Los datos de peso, talla y circunferencia de cráneo de este estudio coinciden con las cifras de estudios extranjeros $^{1,3-9}$, lo cual les otorga mayor va- lidez. No resulta por lo tanto adecuado continuar empleando en nuestro medio las curvas de Denver. Parece recomendable utilizar a nivel nacional una curva o tabla de crecimiento fetal, que para edades gestacionales entre 24 y 34 semanas sería la de este estudio colaborativo y para edades de 35 semanas y más sería la de Juez y cols. ${ }^{12}$.

\section{RESUMEN}

Con el objetivo de tener datos nacionales de peso, talla y circunferencia de cráneo en recién nacidos de 24 a 34 semanas de gestación se realizó un estudio colaborativo restrospectivo de niños nacidos en Santiago, en las Materniđades de los Hospitales José Joaquín Aguirre, del Salvador, Sótero del Río y Clínica AJemana, entre 1982 y 1987 . Se incluyó en el estudio a 379 nifios nacidos de embarazos simples, con edad gestacional confiable y sin patología fetal ni materna de importancia. Se presentan las tablas con promedios y desviaciones estándar de peso, talla y circunferencia craneana, para cada edad gestacional entre 24 y 34 semanas, así como las respectivas curvas con variación de \pm 1,5 desviaciones estándar. Se recomienda el uso de estas tablas y curvas a nivel nacional.

\section{REFERENCIAS}

1. Lubchenco L., Hansman Ch., Dressier M., Boyd $E$.: Intrauterine growth as estimated from liveborn bith-weight data at 24 to 42 weeks of gestation. Pediatrics $1963 ; 32: 793-800$.

2. Lubchenco L., Hansman Ch., Boyd E.: Intrauterime growth in length and head circunference as estimated from live births at gestational ages from 26 to 42 weeks. Pediatrics $1966 ; 37: 403-408$.

3. Babson G., Behrman R., Lessel R.: Liveborn birth weight for gestational age of white middle class infants. Pediatrics $1970 ; 45: 937-944$.

4. Freemon M., Grapes W., Thampson R.: Indigent negro and caucasian birthweight gestational age tables. Pediatrics $1970 ; 45: 9-15$.

5. Sterky G.: Swedish standard curves for intrauterine growth. Pediattics 1970; 46: 7-8.

6. Thompson A., Billewica $W$. . Hytten F.: The assessment of fetal growth. J Obstet Gynecol Brit Cwlth 1986; 75: 903-916.

7. Gruertwald P.: Growth of the human fetus. I. Normal growth and its variation. Am J Obstet and Gynec 1966; Vol 94: 1112-1118.

8. Rantakallo P.: Groups at risk in low birthweight infants and perinatal mortality. Acta Paediatr Scand (Supl) 1931; 58: 1969-1972. 
9. Usher $R_{1}$ Mc Lean $F_{\text {.: }}$ Intrauterine growth of live born caucasian infants at level: Standards obtained from measurements in 7 dimensions of infants born between 25 and 44 weeks of gestation. J Pediatr 1969;74: $901-910$.

10. Neligan $G$.: A comunity study of the relationship between birthweight and gestational age. Clinic Develop Med 1965; 19: 28-32.

11. Guayasamin O., Benedetti $W$., Olthobe O.: Crecimiento fetal humano valorado por irdicadores antropométricos. Bol of Sanit Panam 1976; 481488 .

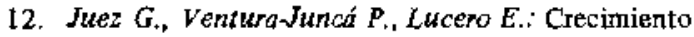
intrauterino en un grupo seleccionado de recién nacidos chilenos. Rev Med Chile 1984; 112: 759-764.

13. Rizzardini $M$., Silvo E. Schldlow D.: Curva de creciniento intrauterino en recién nacidos chilenos. Rev Chil Pediatr 1972; 42: 7-13.
14. Krause S., Grob K., Grob J., Campas G.: Caracter ísticas antropométricas de recién nacidos vivos de Valdivia en comparación con patrón de Battaglia y Lubchenco. Rev Lat Perinatol 1984; 4: 111-118.

15. Naeye R, Dixon J.: Distortions in fetal growth standard. Pedjatr Res 1978; 12: 987-991.

16. Worshaw $J_{.:}$Intrauterine growth setardation: Adaptation or pathology? Pediatrics 1985; 76: 998-999.

17. Hasbún $J$. Sónchez $R$., Cáceres $J$.: La altura y su influencia en las curvas de crecimiento intrauterino en Iecién nacidos chilenos. Rev Obstet Ginecol 1976; 44: 290-297.

18. Ghosh S., Bhargara S., Madhavan S., Tasker A., Nigam S.: Intrauterine growth of north indian babies. Pediatrics $1971 ; 47: 826-830$. 